\section{Successful targeting of mesangial cells using immunoliposomes}

Cell proliferation, secretion of inflammatory cytokines, and production of extracellular matrix by glomerular mesangial cells are implicated in the pathogenesis of lupus glomerulonephritis. Targeting mesangial cells might, therefore, provide an effective treatment strategy for patients with systemic lupus erythematosus. However, identification of a surface marker unique to these cells has so far proved elusive, as has a mode for targeted drug delivery. Immunoliposomes-conjugates of liposomes and antibodies - have now been used to target the surface marker a8 integrin.

Normal and nephritic mice express $\alpha 8$ integrin in their glomeruli. Optimally designed antia8 integrin immunoliposomes loaded with a fluorescent tracking dye (Dil) were injected into the tail vein of $(\mathrm{NZM} \times \mathrm{NOD}) \mathrm{F}_{1}$ mice, a model of spontaneous systemic lupus erythematosus. The anti- $\alpha 8$ integrin immunoliposomes, but not control rabbit IgG immunoliposomes, were detected specifically in glomerular mesangium and mesangial cells in 2-month-old mice (which show no pathologic changes at this age) and in 6-month-old mice (which show signs of mesangial expansion, indicative of acute proliferative glomerulonephritis); the formation of glomerular IgG immune complex deposits in 6-month-old mice did not interfere with this specific targeting.

Trafficking of the Dil-loaded anti- $\alpha 8$ integrin immunoliposomes into other tissues was infrequent and nonspecific and, as human cells also express $a 8$ integrin, targeted delivery by this mode has the potential to provide selective treatment for glomerular diseases in humans.

Original article Scindia Y et al. (2008) Anti-8 integrin immunoliposomes in glomeruli of lupus-susceptible mice: a novel system for delivery of therapeutic agents to the renal glomerulus in systemic lupus erythematosus. Arthritis Rheum 58: 3884-3891

\section{Skin involvement does not predict severe complications of systemic sclerosis}

Skin thickening, usually measured using the modified Rodnan skin score (mRSS), is often used as a surrogate marker for internal organ manifestations of systemic sclerosis (SSc). However, data from 1,200 patients in the German Systemic Sclerosis Network register indicate that a lack of skin sclerosis does not necessarily reflect the absence of severe, life-threatening complications, nor does it indicate early disease in most patients.

Hanitsch et al. reported that a low mRSS $(<3)$ was more often associated with the limited form of SSc and higher mRSS with an increased frequency of the diffuse form of SSc, but that the frequency of serious organ manifestations, including pulmonary arterial hypertension and heart, kidney and musculoskeletal involvement, was similar across the cohort, regardless of mRSS. Other clinical features, such as dyspnea, sicca syndrome and nervous system involvement, were also similarly distributed. Furthermore, disease duration, taken as the first presentation of Raynaud's phenomenon, skin involvement or organ manifestation, did not correlate with mRSS. In accordance with earlier studies, only $61.9 \%$ of patients with an mRSS $<3$ fulfilled the American College of Rheumatology classification criteria for SSc, which focus on fibrosis and sclerosis.

These observations reflect the importance of screening for internal manifestations of SSc, regardless of the degree of external manifestations observed.

Original article Hanitsch LG et al. (2009) Skin sclerosis is only of limited value to identify SSc patients with severe manifestations - an analysis of a distinct patient subgroup of the German Systemic Sclerosis Network (DNSS) Register. Rheumatology (Oxford) 48: 70-73

\section{Hypoxia enhances production of functional cartilage from stem cells}

Engineering cartilage from stem cells is challenging, as several factors must be controlled to ensure that the tissue forms correctly. As cartilage is in a hypoxic environment in vivo, Koay and Athanasiou investigated whether subjecting human embryonic stem cells (hESCs) to hypoxia might benefit the engineering of cartilage in vitro.

The researchers studied chondrogenic differentiation of embryoid bodies over 3 weeks followed by tissue engineering of the differentiated cells over 4 weeks, under either hypoxic conditions $\left(2 \% \mathrm{O}_{2}\right)$ or normoxic conditions $\left(20 \% \mathrm{O}_{2}\right)$ 